\title{
Flavonoid profiling among wild type and related GM wheat varieties
}

\section{Journal Article}

\section{Author(s):}

loset, Jean-Robert; Urbaniak, Bartosz; Ndjoko-loset, Karine; Wirth, Judith; Martin, Frederic; Gruissem, Wilhelm; Hostettmann, Kurt; Sautter, Christof

\section{Publication date:}

2007

\section{Permanent link:}

https://doi.org/10.3929/ethz-b-000007737

\section{Rights / license:}

In Copyright - Non-Commercial Use Permitted

\section{Originally published in:}

Plant Molecular Biology 65(5), https://doi.org/10.1007/s11103-007-9229-9 


\title{
Flavonoid profiling among wild type and related GM wheat varieties
}

\author{
Jean-Robert Ioset - Bartosz Urbaniak · Karine Ndjoko-Ioset · Judith Wirth • \\ Frédéric Martin · Wilhelm Gruissem · Kurt Hostettmann · Christof Sautter
}

Received: 2 February 2007/ Accepted: 17 August 2007/Published online: 12 September 2007

(C) Springer Science+Business Media B.V. 2007

\begin{abstract}
Pleiotropic effects are one of the main concerns regarding genetically modified organisms (GMOs). This includes unintended side effects of the transgene or its genome insertion site on the regulation of other endogenous genes, which could potentially cause the accumulation of different secondary metabolites that may have not only an impact on diet as repeatedly worried by the public but also on the environment. Regarding amount and possible environmental effects, flavonoids represent the most prominent group of secondary metabolites in wheat. Many flavonoids function as signalling or defence molecules. We used a robust and reproducible analytical method to compare the flavonoid content of genetically modified (GM) wheat (Triticum aestivum L., Gramineae) expressing genes that confer increased fungal resistance with their non-GM siblings. The transgenes provide either a broad-spectrum fungal defence (chitinase/glucanase from barley) or bunt-specific resistance by a viral gene (KP4). Significant differences in flavonoid composition were found between different wheat varieties whereas different lines of GM wheat with increased antifungal resistance showed only minor differences in their flavonoid composition relative to their non-GM siblings. In a field test, no significant differences were detectable between infected and non-infected wheat of the same variety regardless of the presence of the transgene. Our results are in agreement with the hypothesis that the transgenes we used to
\end{abstract}

J.-R. Ioset · K. Ndjoko-Ioset · F. Martin · K. Hostettmann Laboratoire de Pharmacognosie et Phytochimie, Ecole Romande de Pharmacie Genève-Lausanne, Université de Genève,

Quai Ernest-Ansermet 30, Geneva 4 1211, Switzerland

B. Urbaniak · J. Wirth · W. Gruissem · C. Sautter ( $₫)$ Institute of Plant Sciences, ETH Zurich, Universitaetsstr. 2, Zurich 8092, Switzerland

e-mail: csautter@ethz.ch increase wheat defence to fungal pathogens do not interfere with the flavonoid biosynthesis pathway. More significantly, the genetic background resulting from conventional breeding has a direct impact on the biological composition of flavonoids, and thus possibly on the environment.

Keywords Flavonoids - Genetic modification · Fungal resistance $\cdot$ Secondary metabolites .

Triticum aestivum . Wheat

$\begin{array}{ll}\text { Abbreviations } \\ \text { APCI } & \text { Atmospheric pressure chemical ionisation } \\ \text { Arb } & \text { Arbitrary units } \\ \text { CID } & \text { Collision induced dissociation energy } \\ \text { DAD } & \text { Diode array detector } \\ \text { ESI } & \text { Electrospray ionisation } \\ \text { GMO } & \text { Genetically modified organism } \\ \text { HPLC/ } & \text { High pressure liquid chromatography w. ultra } \\ \text { UV } & \text { violet detection } \\ \text { HPLC/ } & \text { High pressure liquid chromatography w. mass } \\ \text { MS } & \text { spectrometer } \\ \text { LN } & \text { Liquid nitrogen } \\ \text { PCA } & \text { Principal component analysis } \\ \text { RH } & \text { Relative humidity } \\ \text { RT } & \text { Room temperature } \\ \text { SPE } & \text { Solid phase extraction } \\ \text { Wt } & \text { Wild type }\end{array}$

\section{Introduction}

Concerns have been expressed that genetically modified organisms (GMOs) show pleiotropic and unpredictable 
effects. This includes undesired side effects on other endogenous genes and their regulation caused by the transgene itself or by its insertion site into the genome (Conner and Jacobs 1999). Such effects might include the accumulation of secondary metabolites such as phytoalexins, which are important for plant disease and pest resistance and, therefore, could have an impact on the environment (Harborne 1986; Daniel et al. 1999).

Wheat is one of the most important crops worldwide (source: FAO, http://faostat.fao.org) and fungal diseases are a major factor in losses of wheat harvests, both in terms of quality and quantity (Wiese 1991; Oerke et al. 1994). Many laboratories have produced GM wheat with increased disease resistance or other valuable genetic modifications (Altpeter et al. 1999; Bliffeld et al. 1999; Chen et al. 1999; Clausen et al. 2000; Sivamani et al. 2000; Bieri et al. 2003; Schlaich et al. 2006). Although no GM wheat is commercially produced at present, several attractive applications have already been developed or are currently under development. Based on published reports and our own preliminary HPLC/UV and HPLC/MS experiments, flavonoid derivatives are among the most prominent secondary metabolites present in wheat (Julian et al. 1971; Harborne and Grayer 1993; Adom et al. 2003).

Changes in the accumulation of secondary metabolites might be due to pleiotropic effects of transgenes, but could also be caused by biotic or abiotic stresses. In addition, genetically determined differences in secondary metabolite accumulation between different varieties of a given species can be expected. In order to distinguish statistically significant pleiotropic effects of transgenes on the accumulation of secondary metabolites in GMOs from environmental and variety effects, large numbers of plant samples must be processed using reproducible extraction methods and analysed using sensitive and high-resolution but also robust and reproducible analytical methods. Here we used the optimised method of extraction and detection that meets the required experimental parameters. Leaves have been chosen as a starting material for the extraction, since leaves are the feeding source of many insects and therefore are responsible for many interactions between plant and environment. Changes in the leaf flavonoid composition, even if of minor importance to humans, can severely influence the ecosystem either by destabilising balance between insects and hosts or by influencing plant resistance to biotic and abiotic stresses. Several GM lines and their parental varieties of wheat were compared as well as the results obtained from GM plants and their non-GM siblings. From a field test we compared pathogen infected and non-infected GM wheat (Schlaich et al. 2006). We discuss the implications for the genetic modification of plants.
Table 1 The non-GM/GM wheat varieties/lines used in this study

\begin{tabular}{lllll}
\hline Variety & wt & Chit/Gluc & RIP & KP4 \\
\hline Frisal & + & + & + & \\
Golin & + & & & + \\
Greina & + & & & + \\
\hline
\end{tabular}

Frisal, Golin and Greina GM wheat lines expressing barley $\beta-1,3-$ glucanase (Chit/Gluc) (Bieri et al. 2000), ribosome-inactivating protein (RIP) (Bieri et al. 2003) and antifungal protein (KP4) (Clausen et al. 2000) were studied together with their parental varieties (wt). Transgenes used are marked with "+”

\section{Materials and methods}

\section{Plant material}

Wheat plants (Triticum aestivum L., Gramineae) of the Swiss spring varieties Frisal, Golin and Greina were transformed with antifungal genes of broad-spectrum effect like chitinase and glucanase (Bieri et al. 2003), ribosome-inactivating protein (RIP) (Bliffeld et al. 1999; Bieri et al. 2000), as well as with a transgene expressing KP4, which is active against smuts and bunts (Clausen et al. 2000). Plants were transformed as described before (Bliffeld et al. 1999) using a Particle Inflow Gun (Finer et al. 1992). In all cases, homozygotic T4 generations were used for the comparisons. A compilation of the plant lines used can be found in Table 1 .

Plants were grown in greenhouse conditions under a 15-h photoperiod at $12-15^{\circ} \mathrm{C}$ and $60 \%$ relative humidity (RH) during the night, and $16-19^{\circ} \mathrm{C}$ and $60 \% \mathrm{RH}$ during the day. Flag leaves of 10 plants were collected and pooled in one sample at the three-leaf stage (developmental stage 13 according to Zadoks et al. 1974). After collection, leaves were frozen in liquid nitrogen $\left(\mathrm{LN}_{2}\right)$ and subsequently stored at $-80^{\circ} \mathrm{C}$ before extraction. All plants were grown together under identical conditions. Leaves were collected always at the same time of the day and the collection window was kept as short as possible (max. $1 \mathrm{~h}$ ) to avoid diurnal variability of the samples.

\section{Extraction of flavonoids}

Samples for analysis were obtained using a solid phase extraction (SPE) technique on $\mathrm{C} 18$ Chromabond 6-ml cartridges (Macherey-Nagel, CH-4702 Oensingen, Switzerland). Before extraction, the plant material was ground in $\mathrm{LN}_{2}$ for maximum $30 \mathrm{sec}$ to obtain a fine powder. SPE cartridges were conditioned with $5 \mathrm{ml}$ of $70 \% \mathrm{CH}_{3} \mathrm{CN}$ (Romil 230 gradient quality, Amman-Technik AG, CH5742 Köliken, Switzerland). An amount of $500 \mathrm{mg}$ of frozen material was placed in the cartridge together with $1 \mathrm{ml}$ of apigenin 7-O-glucoside (Carl Roth $\mathrm{GmbH}+\mathrm{Co}$, 
D-76185 Karlsruhe, Germany) $\mathrm{MeOH}$ solution as an internal standard $(0.35 \mathrm{mg} / \mathrm{ml})$. During extraction, two fractions were collected: fraction $\mathrm{A}$ was extracted with $10 \mathrm{ml}$ of $70 \% \mathrm{CH}_{3} \mathrm{CN}$ followed by $10 \mathrm{ml}$ of $100 \%$ $\mathrm{CH}_{3} \mathrm{CN}$; fraction B was eluted with $10 \mathrm{ml}$ of $100 \%$ $\mathrm{CH}_{3} \mathrm{CN}$. Fraction B served as a control, for quantitative extraction of the compounds of interest, since all secondary metabolites to be detected were expected in fraction A. All extraction steps were performed at RT. After extraction, samples were evaporated to dryness using a Rotavapor (Büchi, CH-9230 Flawil, Switzerland) and lyophilised with a Gamma 1-20 lyophiliser (Christ, Blanc-Labo SA, CH-1131 Tolochenaz-Morges, Switzerland). Extraction of each batch of leaves was repeated three times and each extract was divided into two aliquots before the analysis.

\section{HPLC/DAD-UV analysis}

Reversed-phase HPLC was carried out using a HPLC system HP-1050 (Hewlett-Packard, Palo Alto, Calif., USA) equipped with a binary pump and Hewlett-Packard (D-76337 Waldbronn, Germany) 1050 series on-line photodiode array detector (DAD). This instrumentation was controlled by HP Chemstation software. The separation was performed on a C-18 Symmetry column $(250 \times 4.6 \mathrm{~mm}$ i.d., 5- $\mu \mathrm{m}$ particle size, Bedford, Mass., USA) with a $\mathrm{MeOH}$ (0.5\% TFA): $\mathrm{H}_{2} \mathrm{O}$ (0.05\% TFA) stepped gradient $26: 74$ to 26:74 in $10 \mathrm{~min}, 26: 74$ to $30: 70$ in $10 \mathrm{~min}, 30: 70$ to $30: 70$ in $20 \mathrm{~min}, 30: 70$ to $100: 0$ in $25 \mathrm{~min}$ and 100:0 to $100: 0$ in $5 \mathrm{~min}$. The column temperature was set at $25^{\circ} \mathrm{C}$ with a flow of $1 \mathrm{ml} / \mathrm{min}$. The DAD-UV detector was set at $271 \mathrm{~nm}$ as a common $\lambda$ max shared by all detected compounds to avoid any variation in terms of spectrophotometric detection that could be induced by the selection of a non- $\lambda$ max value. HPLC/DAD-UV analyses were performed in duplicate by injection of a $20 \mu \mathrm{l}$ volume of fraction A samples and, for control fraction B samples $(10 \mathrm{mg} / \mathrm{ml})$.

LC/DAD-UV analysis with post-column addition of UV-shift reagents

The method used for post-column addition of UV-shift reagents is based on a previously reported protocol (Wolfender and Hostettmann, 1993).

\section{HPLC/MS-MS analysis}

The HPLC conditions described above were used for the HPLC separation but $0.1 \%$ formic acid (Fluka Chemie GmbH, CH-9471 Buchs, Switzerland) was added instead of
TFA to both HPLC solvents. HPLC/MS spectra were recorded on a Finnigan LCQ ion trap mass spectrometer (FinniganMAT, San Jose, Calif., USA). The ionisation was obtained using both atmospheric pressure chemical ionisation (APCI) and electrospray ionisation (ESI) sources. The APCI optimised parameters were: capillary temperature $150^{\circ} \mathrm{C}$, vaporizer temperature $450^{\circ} \mathrm{C}$, corona needle current $4.5 \mu \mathrm{A}$ and sheath gas flow 65 arbitrary units (arb). Spectra (160-900 amu) were recorded in the positive ion mode. MS-MS experiments (collision energy (CID) of $40 \%$, isolation width of $2 \mathrm{amu}$ ) were performed after selection and consecutive fragmentation of the most intense ion of the MS. Isoorientin used as a reference compound was previously isolated in our laboratory from Gentiana lutea L. (Gentianaceae) and fully characterized. The purity of isolated compound, checked by HPLC/DAD-UV was $>95 \%$.

In MS-MS analysis using ESI in negative mode, the parent ions were also selected for MS-MS experiments. Sheath gas flow rate was set at $70 \mathrm{arb} \% \%$, the spray needle at $2.5 \mathrm{kV}$. The voltage and the temperature of the heated capillary were $4.7 \mathrm{kV}$ and $200^{\circ} \mathrm{C}$, respectively. Source induced dissociation energy (SID) was used at $15 \%$. CID was adjusted to $100 \%$ (isolation width of $2 \mathrm{amu}$ ) for fragmentation experiments in the trap using helium as the collision gas. The fragment ions were denoted according to the nomenclature proposed by Domon and Costello (1988).

\section{Data processing}

Following the HPLC separation, the $271 \mathrm{~nm}$ UV chromatograms were integrated and normalized to the internal standard present in each sample with ACD Chrom Processor software (http://www.acdlabs.com). To test the significance of changes, analysis of variance (ANOVA) and t-test were used. In addition, we applied the hierarchical clustering (Eisen et al. 1998; Guess and Wilson 2002) with unweighted pair-group average linkage method and principal component analysis (Raychaudhuri et al. 2000) to represent similarities between analysed samples. Distances between each sample were measured using the Euclidian distance function. Concentrations of the compounds were $\log$ transformed before calculation to avoid the influence of highly abundant compounds on the results. Clustering was performed with R package (http://www. r-project.org) and all other statistical calculations were performed using the SPSS software pack (http://www.spss.com).

Results and discussion

Preliminary HPLC/DAD-UV and HPLC/MS experiments performed on $\mathrm{MeOH}$ and $\mathrm{CH}_{3} \mathrm{CN}$ extracts of wheat leaves 
established the presence of flavonoids in these extracts. Indeed, the UV spectra of major detected compounds showed the characteristic pattern of flavones with the presence of two maxima located around 270 and $350 \mathrm{~nm}$. We focused our analyses on these compounds because they have important functions in ecological systems as one of the major classes of secondary metabolites found in wheat (Julian et al. 1971; Harborne and Grayer 1993; Adom et al. 2003). Significant changes in the wheat flavonoid profile could have an impact on the plant environment (Harborne 1986; Daniel et al. 1999).

\section{Adaptation of the method}

Flavonoids were selectively extracted from the plant material using a one-step solid phase extraction (SPE) with a $10-\mathrm{ml}$ volume of a $70 \% \mathrm{CH}_{3} \mathrm{CN}$ solution (fraction $\mathrm{A}$ ). To reduce individual sample variation, leaves from 10 plants growing under identical conditions were harvested and pooled before extraction. The control fraction $\mathrm{B}$ (wash with $100 \% \mathrm{CH}_{3} \mathrm{CN}$ ) contained no flavonoids, indicating a quantitative extraction of these compounds in the first step (fraction A). Fractions A from the different GM wheat plants and their non-GM siblings (GM/non-GM) were analysed by both HPLC/DAD-UV and HPLC/MS. An example of a typical chromatographic profile recorded by UV spectrophotometry at $271 \mathrm{~nm}$ is shown in Fig. 1. The comparison of different wheat lines described below was based on the analysis of profiles from such chromatograms. Extraction of each batch of leaves was repeated three times and each extract was divided into two aliquots before analysis. Very low technical variability (determined by analysis of the same batch of leaves) compared with measured biological differences between samples (RSD of 0.059 and 0.181 , respectively) confirmed this method as suitable for comparative purposes.

\section{Identification of the detected flavonoids}

On the basis of previous studies that reported the presence of both $O$ - and $C$-glycosylated flavonoids in wheat leaves (Harborne and Hall 1964; Julian et al. 1971; Wagner et al. 1980), reversed-phase HPLC combined with UV and mass spectrometric detection (LC/DAD-UV/MS) under the

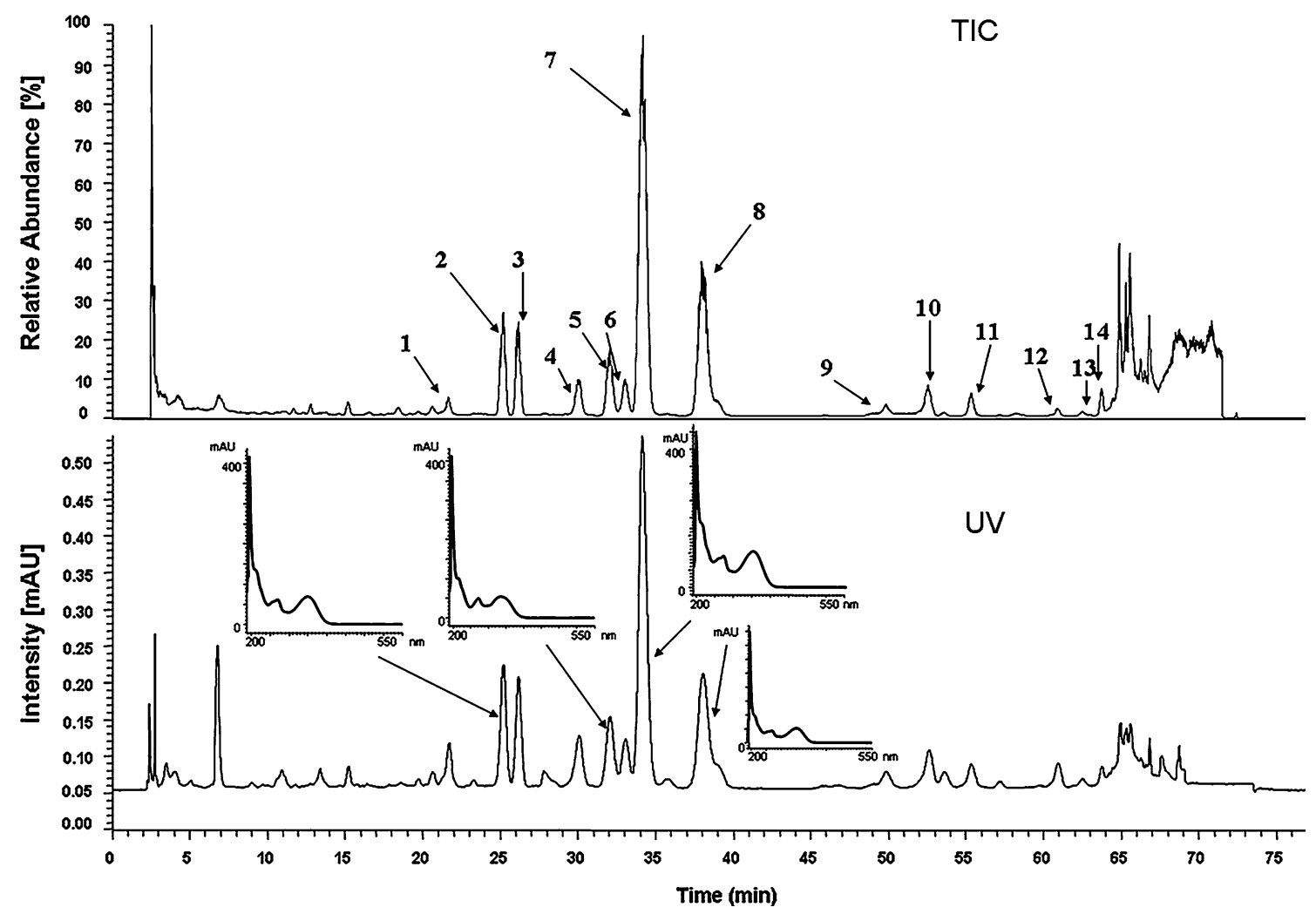

Fig. 1 HPLC/UV/APCI-MS of a sample of Greina wt. TIC: Total ion current; UV-ultraviolet absorption spectrum. Conditions of separation: C-18 Symmetry $\AA$ column, $\mathrm{MeOH}\left(0.5 \%\right.$ formic acid): $\mathrm{H}_{2} \mathrm{O}$ (0.5\% formic acid) gradient $10: 90$ to $26: 74$ in $10 \mathrm{~min}, 26: 74$ to $30: 70$ in $10 \mathrm{~min}, 30: 70$ to $30: 70$ in $20 \mathrm{~min}, 30: 70$ to $100: 0$ in $25 \mathrm{~min}$ and 100:0 to $100: 0$ in $5 \mathrm{~min}$. Column temperature: $25^{\circ} \mathrm{C}$. Flow: $1 \mathrm{ml} / \mathrm{min}$. UV detection: $271 \mathrm{~nm}$. Inlet squares show UV spectra of selected compounds 
ionisation conditions optimised by Waridel et al. (2001) was chosen to analyse fraction A samples (see Results and Discussion, Adaptation of the method) of the Greina wt variety. This LC/DAD-UV/MS method has the advantage that it distinguishes between $C$-glycoside flavonoids and $O$-glycoside analogues based on specific MS/MS fragmentation. Detected peaks were numbered according to their elution time (Fig. 1). All peaks exhibited a UV spectrum specific for flavonoid derivatives with two major absorption bands: I around $\lambda \max 350 \mathrm{~nm}$ and II at $\lambda \max 270 \mathrm{~nm}$ (Fig. 1). The major detected peak (7) was identified as isoorientin after LC/UV and LC/MS/MS comparison with the reference compound (Table 2). The fragmentation of the parent ion at $m / z, 449[\mathrm{M}+\mathrm{H}]^{+}$shown in Fig. 2 led to the observation of specific cleavages for $C$-glycosides in $\mathrm{MS}^{2}$ with characteristic ions at $\mathrm{m} / \mathrm{z}, 431[\mathrm{M}+\mathrm{H}-18]^{+}, 413$ $[\mathrm{M}+\mathrm{H}-36]^{+}, 395[\mathrm{M}+\mathrm{H}-54]^{+}, 383[\mathrm{M}+\mathrm{H}-30-36]^{+}, 353$ $[\mathrm{M}+\mathrm{H}-96]^{+}$and $329[\mathrm{M}+\mathrm{H}-120]^{+}$. The presence of the ${ }^{0,2} \mathrm{X}^{+}$fragment at $\mathrm{m} / \mathrm{z} 329[\mathrm{M}+\mathrm{H}-120]^{+}$is of particular importance as it allows unambiguous identification of the 6$O$-glycoside flavonoid isoorientin from its 8 - $O$-glycoside analogue orientin (Waridel et al. 2001). This observation was confirmed by injection of the standard substance orientin using the same analytical conditions (not shown).

This LC/DAD-UV/MS method was used to draw similar structural conclusions about compounds 1-14. The assumed chemical identities of the flavonoids detected in fraction A obtained from the Greina wt sample are presented in Table 2. These identities have been concluded from the LC/UV/MS and $\mathrm{MS}^{2}$ analyses in complement with data from post-column addition of UV-shift reagents. The shifts observed were interpreted according to the rules previously established for the analysis of pure polyphenols
(Markham 1982). A shift of band II was observed (5-14 nm) on the NaOAc spectrum, suggesting the presence of a free $4^{\prime}-\mathrm{OH}$ substitution for all compounds. This was confirmed by measurement of a shift of band I between 45 and $65 \mathrm{~nm}$ in the $\mathrm{KOH}$ spectrum. The $\mathrm{H}_{3} \mathrm{BO}_{3} / \mathrm{NaOAc}$ spectra indicated that all compounds listed in Table 2, except 4 and 6, showed a O-diOH substitution on cycle B due to the presence of a shift of at least $10 \mathrm{~nm}$ of band I. This was confirmed by the absence of a shift of band I between the $\mathrm{AlCl}_{3}$ and $\mathrm{AlCl}_{3} / \mathrm{HCl}$ spectra of $\mathbf{4}$ and $\mathbf{6}$.

Finally, a shift of $56 \mathrm{~nm}$ or more on band I $(37 \mathrm{~nm}$ for 4 and 6) was recorded for all compounds, indicating the absence of a free 3-OH group and the presence of a chelated $5-\mathrm{OH}$ substituent on the aglycone. No conclusion could be drawn about the presence of a 7-OH free substitution, as the expected shift $(5-20 \mathrm{~nm})$ was not observed with the reference compound isoorientin in the $\mathrm{NaOAC}$ spectrum.

An extensive study of MS/MS fragments of di- $O$-flavonols was published by Cuyckens and Claeys (2004) and by Ferreres et al. (2004). Some conclusions on the interglycosidic linkage were drawn according to the relative intensities of fragments observed. Hypothesis on the interglycosidic linkage of compounds $\mathbf{1}$ and $\mathbf{8}$ was attempted using the same methodology. With the LCQ, stable and reproducible fragments were only observed with a high collision energy of $100 \%$. Compounds $\mathbf{1}$ and $\mathbf{8}$ exhibited both ${ }^{0,2} \mathrm{X}_{0} \mathrm{Y}_{1}^{-}$as base peaks corresponding to the loss of two units of the molecular weight 162 and 120. In di-O-glycosyl flavonols, the occurrence of this fragment as base peak is related to $1 \rightarrow 2$ linkage. Based on this consideration, a $1 \rightarrow 2$ linkage could be suggested for compounds 1 and $\mathbf{8}$. The positions of the sugars in the pairs of isomers at $\mathrm{m} / z 579[\mathrm{M}-\mathrm{H}]^{-}(\mathbf{2} / \mathbf{3})$ and $\mathrm{m} / z 563$

Table 2 Chemical identity of the major UV-detected constituents of a wt Greina extract and their MS and MS ${ }^{2}$ fragmentation patterns with their respective abundance given in brackets

\begin{tabular}{|c|c|c|c|}
\hline No & Name & {$[\mathrm{M}+\mathrm{H}]$} & $\mathrm{MS}^{2}$ \\
\hline 1 & 6- $O^{\prime \prime}$-hexosyl isoorientin & 611 & 449 [21]; 431 [25]; 383 [6]; 329 [12]; 299 [1] \\
\hline 2 & 8- $C$-pentosyl-6- $C$-hexosyl luteolin & 581 & $563[40] ; 527[26] ; 497[8] ; 461[5] ; 443[33]$ \\
\hline 3 & $8-C$ - hexosyl $-6-C$-pentosyl luteolin & 581 & $563[65] ; 497[25] ; 461[14]$ \\
\hline 4 & 8- $C$ - hexosyl -6- $C$-pentosyl apigenin & 565 & 547 [23]; 529 [34]; 511 [23]; 427 [27]; 409 [10] \\
\hline 5 & 7- $O$-hexosyl isoorientin & 611 & 449 [62]; 431[25]; 413 [5]; 383 [10]; 353 [7]; 329 [5] \\
\hline 6 & 8-C-pentosyl-6- $C$-hexosyl apigenin & 565 & 547 [67]; 529 [19]; 499 [28]; 469 [13]; 457 [12]; 445 [16] \\
\hline 7 & isoorientin (reference standard) & 449 & 431 [20]; 383 [30]; 353 [15]; 329 [13]; 299 [2] \\
\hline 8 & $6^{\prime \prime}-O$-pentosyl isoorientin & 595 & 449 [97]; $431[20] ; 383$ [5]; 329 [4] \\
\hline 9 & methoxylated $O$-hexosyl isoorientin & 625 & 463 [52]; 445 [15]; 427 [5]; 397 [7]; 367 [3] \\
\hline 10 & 6-hexosyl chrysoeriol & 463 & 445 [66]; 427 [27]; 409 [11]; 397 [45]; 367 [26]; 343 [12]; 313 [5] \\
\hline 11 & methoxylated $O$-pentosyl isoorientin & 609 & 463 [90]; 445 [22]; 397 [5]; 367 [1]; 343 [6]; 313 [1] \\
\hline 12 & apigenin-7-glucoside (int. standard) & 433 & not analyzed by MS/MS \\
\hline 13 & $O$-hexosyl- $O$-pentosyl tricin & 639 & 493 [NA]; $331[\mathrm{NA}]$ \\
\hline 14 & $O$-hexosyl tricin & 493 & $331[\mathrm{NA}]$ \\
\hline
\end{tabular}

NA not available 


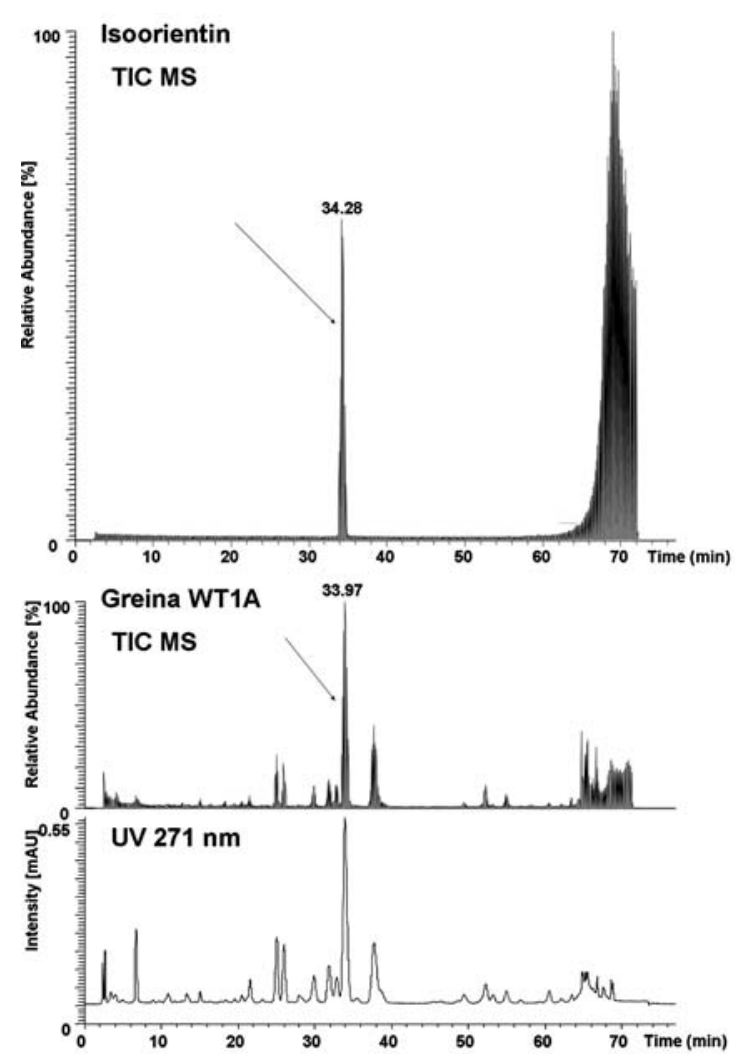

Fig. 2 Identification of isoorientin by HPLC combined with UV and Atmospheric Pressure Chemical Ionisation Mass Spectrography (HPLC/UV/APCI-MS) in a sample of Greina wt. Conditions of separation: C-18 Symmetry $\AA$ column, $\mathrm{MeOH}(0.5 \%$ formic acid):

$[\mathrm{M}-\mathrm{H}]^{-}(\mathbf{4} / \mathbf{6})$ were assigned considering the study on lucenin 1/lucenin 3 and isoschaftoside/schaftoside led by Ferreres et al. (2003). Based on the MS/MS spectra recorded, 2 and $\mathbf{3}$ were assumed to be 8- $C$-pentosyl-6- $C$-hexosyl luteolin and 8-C- hexosyl -6- $C$-pentosyl luteolin, respectively. 8- $C$-pentosyl-6- $C$-hexosyl apigenin was attributed to 6 and 4 to $8-C$ - hexosyl -6- $C$-pentosyl apigenin.

Compound 5 was comparable to 7-O-hexosyl isoorientin. The departure of the $O$-hexosyl moeity was recorded at a relative abundance of $100 \%$ at $\mathrm{m} / z \quad 429\left(\begin{array}{lll}-180 & \mathrm{u}\end{array}\right)$. Cleavage of the $C$ - hexosyl unit was observed at $\mathrm{m} / z 327$ $(-180-120 \mathrm{u})$ with a relative abundance of $100 \%$. Compared with the spectrum of compound $\mathbf{8}$ and with its $(1 \rightarrow 6)$ analogue, the fragmentation pattern observed for $\mathbf{5}$ was completely different, suggesting that the glycoside moieties were located at two distinct positions (not shown).

\section{Comparison of different wheat lines}

In order to compare different GM/non-GM wheat varieties objectively, a hierarchical clustering (Eisen et al. 1998; Guess and Wilson 2002) using an unweighted pair-group

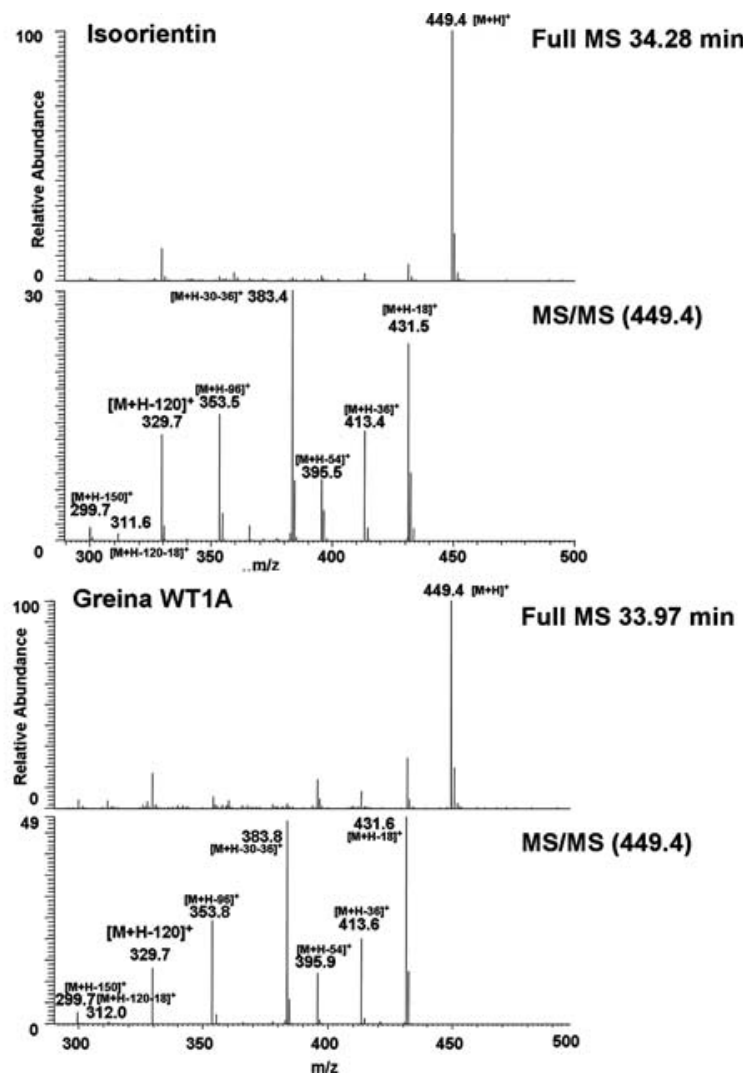

$\mathrm{H}_{2} \mathrm{O}(0.5 \%$ formic acid) gradient $10: 90$ to $26: 74$ in $10 \mathrm{~min}, 26: 74$ to $30: 70$ in $10 \mathrm{~min}, 30: 70$ to $30: 70$ in $20 \mathrm{~min}, 30: 70$ to $100: 0$ in $25 \mathrm{~min}$ and 100:0 to $100: 0$ in $5 \mathrm{~min}$. Column temperature: $25^{\circ} \mathrm{C}$. Flow: $1 \mathrm{ml} /$ min. UV detection: $271 \mathrm{~nm}$

average linkage method was performed on the relative fingerprinting data obtained from HPLC/UV analyses at $271 \mathrm{~nm}$. Peaks were aligned manually using ACD Chrom Processor 7 software. Individual peak values were $\log$ transformed and distances between samples were calculated using the Euclidean distance function (Claverie 1999). The most related sample pairs clustered together (Fig. 3). Bootstrap resampling (Shimodaira 2004) was used to evaluate the accuracy of the clustering. The numbers on the graph represent $P$-values calculated by the bootstrapping algorithm and are a measure of cluster relevance. These show the relative frequency (in percent) at which a particular cluster appeared during multiple cluster analysis. The length of the horizontal lines represents the similarity of the samples: the longer the line, the greater the variation between samples. The method revealed a closer correlation between GM/non-GM plants of the same variety than between wt plants of different varieties.

The significance of the changes was confirmed by analysis of variance (ANOVA) and two-tail $t$-test. Intravariety changes were calculated by $t$-test, since samples are grouped in natural pairs, i.e. GM/non-GM in relevant varieties. In contrast, inter-variety changes were analysed 
Fig. 3 Hierarchical clustering of the samples. The lengths of the vertical lines represent the distances between samples. Numbers next to the nodes represent $P$-values (in \%) computed by the bootstrap resampling algorithm

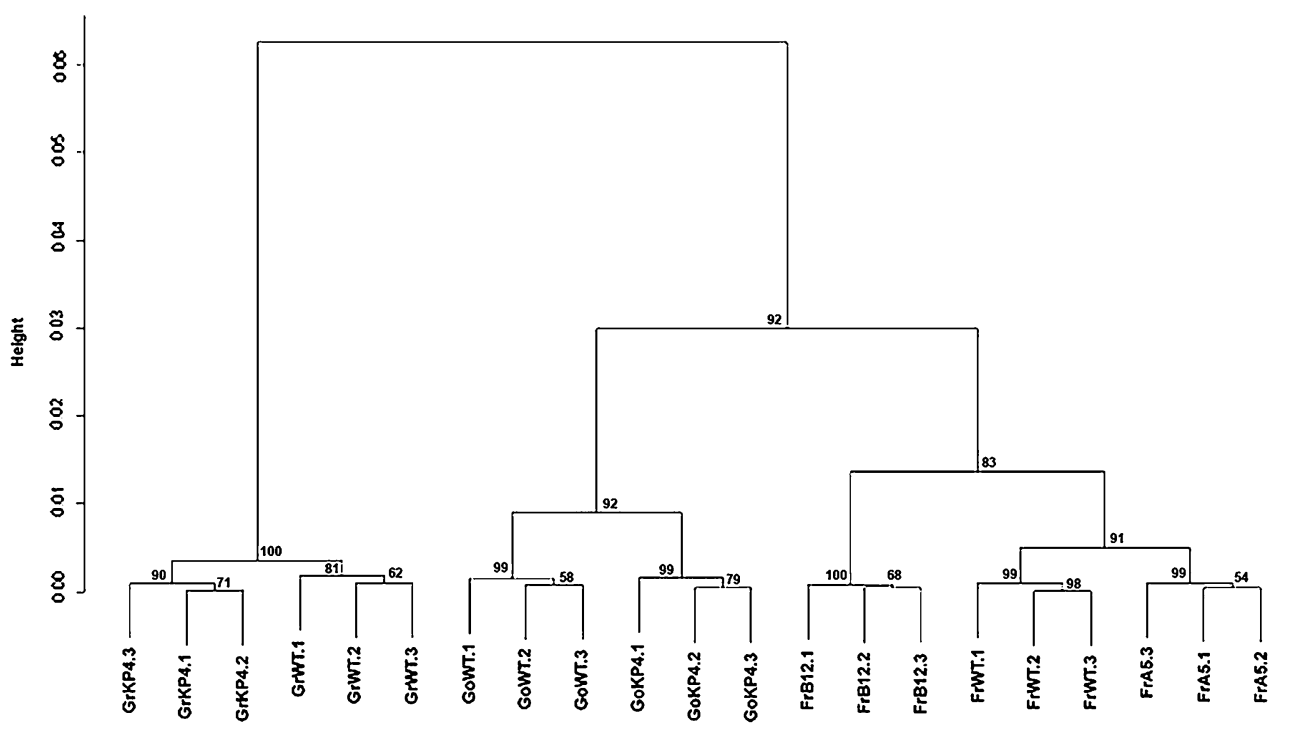

by the ANOVA test, since it enables stricter significance levels than the multiple t-test for a larger number of groups. All calculations were performed for $P=0.01$ and the results are presented as histograms (Fig. 4). Any differences in intra-variety GM/non-GM comparisons were in all cases significantly smaller (if not undetectable) than the differences occurring between wt plants of different varieties.

To obtain a synoptic view of the comparison, we used principal component analysis (PCA) according to Raychaudhuri et al. (2000). This reduced the number of factors responsible for variation between the samples to three main components carrying the $88.5 \%$ of sampleto-sample variability. The PCA begins by searching for a linear combination of variables that accounts for as much variation in the original variables as possible. It then searches for a further component that accounts for as much of the remaining variation as possible and which is uncorrelated with the previous component. This is continued until there are as many components as original variables. Usually just a few components account for most of the variation and these components are used to replace the original variables. After calculation, three main components proved to carry $88.5 \%$ of sample-to-sample variability. Table 2 shows that the main factors correlated with component 1 are peaks $\mathbf{6 , 8}$ and 11. Component 2 is correlated with peaks 3 and $\mathbf{1 4}$ and component 3 with peaks 1 and 4. After plotting in the 2-dimensional space, components 1 and 2 carrying $74.5 \%$ of sample-to-sample variability allowed the separation of samples into clusters where the division between varieties is clearly visible (Fig. 5). Moreover, PCA also showed that component 1 allows for a separation between varieties tested. Therefore, it can be concluded that the main differences between those varieties are carried by compounds designated by three peaks: 6, 8 and $\mathbf{1 1}$ (see Table 3).
As it was detectable between conventional varieties than between GM lines and their wt parents (see Figs. 3-5). Furthermore, even between varieties, only quantitative differences in compound concentrations were observed and there were no qualitative changes in the overall profile patterns.

As the second approach we compared the spring wheat variety Greina with its KP4-transformed GM line. The KP4 is a viral gene and its secreted protein has antifungal activity specifically against fungal species of the order Ustilaginales, vulgo smuts and bunts (Clausen et al. 2000). We infected these plants with stinking smut (Tilletia caries) in a field test, which was performed in 2004 (Schlaich et al. 2006). The treatments in the fieldtests were:

Table 3 PCA rotated component matrix

\begin{tabular}{lrrr}
\hline & \multicolumn{2}{l}{ Component } & \multicolumn{1}{l}{3} \\
\cline { 2 - 4 } & \multicolumn{1}{l}{ 1 } & \multicolumn{2}{l}{} \\
\hline Peak1 & .317 & .194 & .822 \\
Peak2 & .573 & .676 & .427 \\
Peak3 & -.055 & .950 & -.235 \\
Peak4 & -.188 & -.230 & .869 \\
Peak5 & .795 & .455 & .299 \\
Peak6 & -.935 & .082 & -.034 \\
Peak7 & .711 & .665 & .164 \\
Peak8 & -.927 & -.009 & .205 \\
Peak9 & .651 & .528 & .300 \\
Peak10 & .684 & .593 & .280 \\
Peak11 & -.947 & -.166 & .131 \\
Peak13 & -.219 & .575 & .572 \\
Peak14 & .273 & .906 & .044 \\
\hline
\end{tabular}

Extraction method: Principal component analysis

Rotation method: Varimax with Kaiser normalization

Rotation converged in 4 iterations 

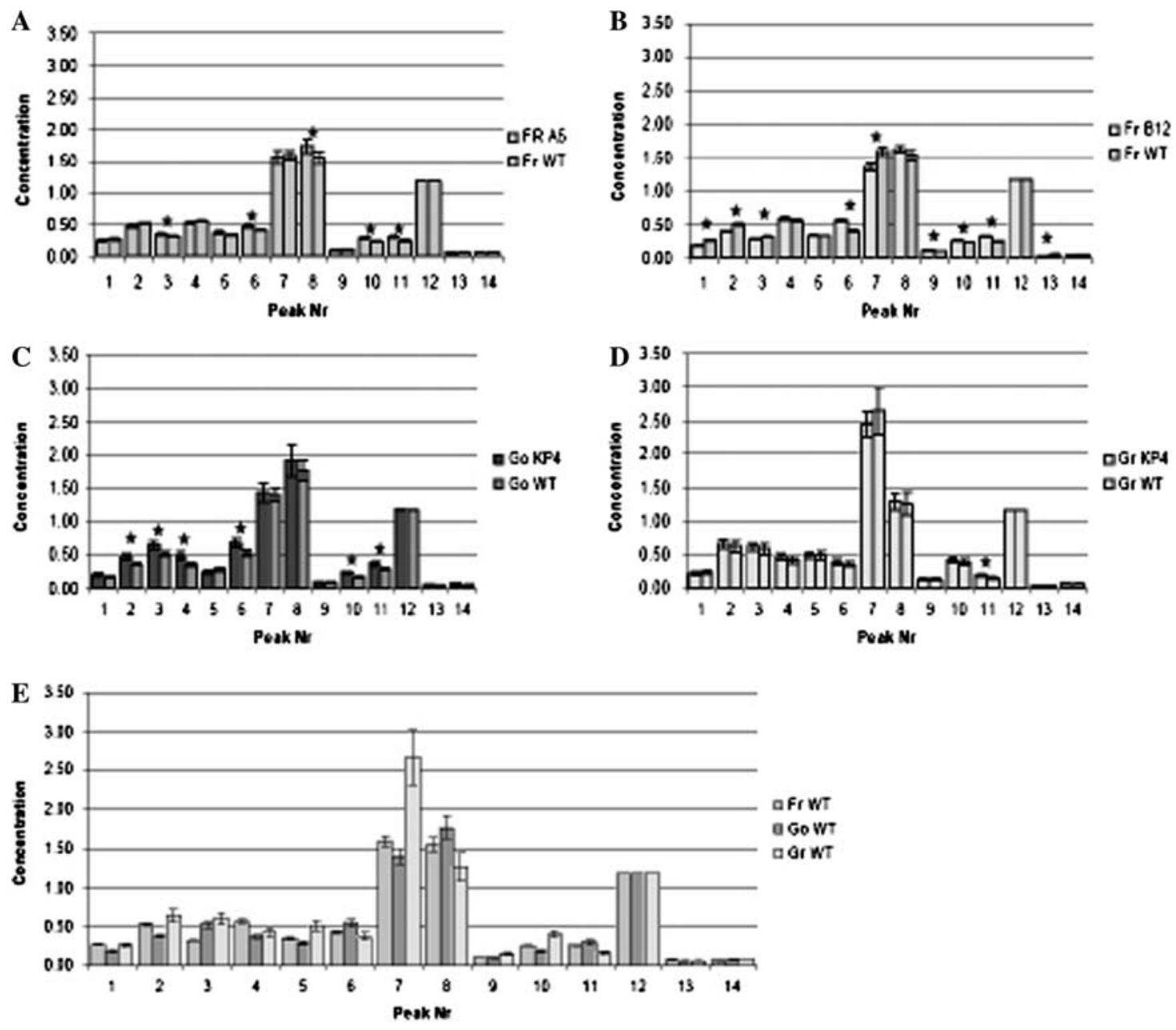

Fig. 4 Inter- and intra-variety sample comparison. Peaks are plotted against an arbitrary concentration value that was normalized over all samples. (A-D) Divalent comparisons between GM lines and the wt

GM line, pathogen-infected and non infected versus the null segregate which lost the transgen by Mendelian segregation, also infected and non infected. In addition we

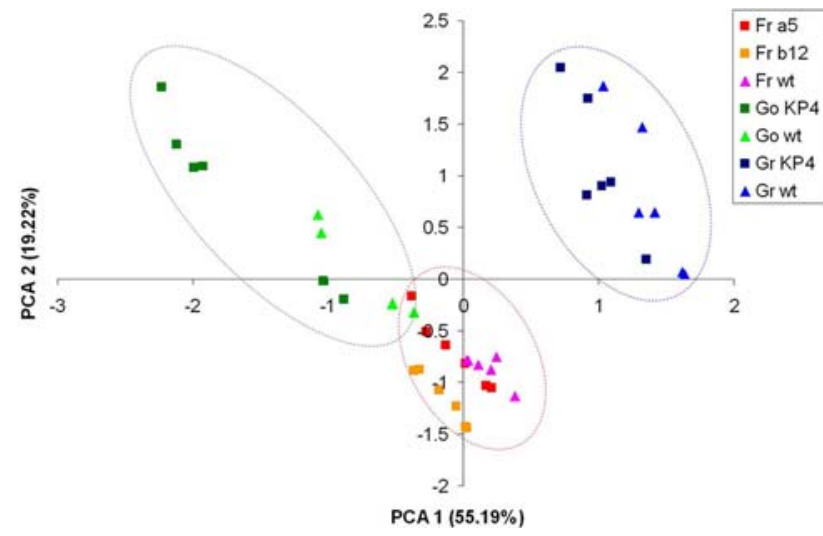

Fig. 5 Principal component analysis. Principal component analysis shows clustering of different varieties together with respective GM lines. Separated clusters are marked with dashed lines varieties. * significant differences between samples at the $P=0.01$. (E) Trivalent comparison of 3 wild-type lines; significant differences were observed between all peaks at $P=0.01$

treated the pathogen infected null segregate with fungicide. (for further details see Schlaich et al. 2006). For analysis of the flavonoid content three biological replicates have been tested. No significant differences in flavonoid content were detectable between the GM line and its null segregate, nor between infected or not infected plants or the fungicide treatment (not shown).

We hypothesise that the only parameter showing detectable differences would have been the genetic background of the wt variety. However only one single variety was tested for its flavonoid content during that field experiment. Observed results are consistent with the insect scoring in that field study of KP4-wheat. We found significant differences in insect infestation between the varieties, but no difference between all other parameters, i.e. GM line and null segregate or pathogen infection (Schlaich et al. 2006, supplementary material). In other words: we found no difference in insect infestation when we also found no differences in flavonoid content. 
The published studies observed similar results in different GM plants, like potato metabolome (Catchpole et al. 2005) and proteome (Lehesranta et al. 2005), tomato (Corpillo et al. 2004), or transcriptome and metabolome of GM wheat (Baker et al. 2006; Baudo et al. 2006). Complex analyses of glyphosate tolerant wheat MON 71800 found no significant changes in 88 different components compared with wt plants (Obert et al. 2004). Comparative analyses of different GM plants regarding substantial equivalence have been reviewed by Cellini et al. (2004). However substantial equivalency is not always granted: unintended effects of conventional breeding as well as of genetic modification have been reported for particular traits (Cellini et al. 2004). For example, low yields of powdery mildew-resistant barley and pest-resistant potato (Harvey et al. 1985; Thomas et al. 1998), a high content of furanocoumarins in pest-resistant celery (Beier 1990), necrotic lesions in wheat expressing phosphatidyl serine synthase (Delhaize et al. 1999), and multiple metabolic changes in canola overexpressing phytoene synthase (Shewmaker et al. 1999).

\section{Conclusions}

We used a rapid and robust approach for the analysis of the flavonoid profile of GM wheat lines and their parental varieties and in addition also GM lines grown in the field where they were exposed to fungal infection. We found significant differences between the non-GM varieties, but not between any other parameters or genotypes. These results correlate with the insect scoring from the field test, indicating that the analysed transgenes have no detectable effect on the flavonoid profile or the ecosystem.

Acknowledgements We would like to thank Elke Fenner for technical assistance, Katalin Konya and Sabine Klarer for the propagation and maintenance of our plants in the greenhouse and Catherine Werlen for help during the initial stage of the experiments.

\section{References}

Adom KK, Sorrells ME, Liu RH (2003) Phytochemical profiles and antioxidant activity of wheat varieties. J Agric Food Chem 51:7825-7834

Altpeter F, Diaz I, McAuslane $\mathrm{H}$ et al (1999) Increased insect resistance in transgenic wheat stably expressing trypsin inhibitor CMe. Mol Breed 5:53-63

Baker JM, Hawkins ND, Ward JL et al (2006) A metabolomic study of substantial equivalence of field-grown genetically modified wheat. Plant Biotechnol J 4:381-392

Baudo MM, Lyons R, Powers S et al (2006) Transgenesis has less impact on the transcriptome of wheat than conventional breeding. Plant Biotechnol J 4:369-380
Beier RC (1990) Natural pesticides and bioactive components in foods. Rev Environ Contam Toxicol 113:47-137

Bieri S, Potrykus I, Fütterer J (2000) Expression of active barley seed ribosome-inactivating protein in transgenic wheat. Theor Appl Genet 100:755-763

Bieri S, Potrykus I, Fütterer J (2003) Effects of combined expression of antifungal barley seed proteins in transgenic wheat on powdery mildew infection. Mol Breed 11:37-48

Bliffeld M, Mundy J, Potrykus I (1999) Genetic engineering of wheat for increased resistance to powdery mildew disease. Theor Appl Genet 98:1079-1086

Catchpole GS, Beckmann M, Enot DP et al (2005) Hierarchical metabolomics demonstrates substantial compositional similarity between genetically modified and conventional potato crops. Proc Natl Acad Sci USA 102:14458-14462

Cellini F, Chesson A, Colquhoun I et al (2004) Unintended effects and their detection in genetically modified crops. Food Chem Toxicol 42:1089-125

Chen WP, Chen PD, Liu DJ (1999) Development of wheat scab symptoms is delayed in transgenic wheat plants that constitutively express a rice thaumatin-like protein gene. Theor Appl Genet 99:755-760

Clausen M, Krauter R, Schachermayr G et al (2000) Antifungal activity of a virally encoded gene in transgenic wheat. Nat Biotechnol 18:446-449

Claverie JM (1999) Computational methods for the identification of differential and coordinated gene expression. Hum Mol Genet 8:1821-1832

Conner AJ, Jacobs JME (1999) Genetic engineering of crops as potential source of genetic hazard in the human diet. Mutat Res Genet Toxicol and Environ Mutagen 443:223-234

Corpillo D, Gardini G, Vaira AM et al (2004) Proteomics as a tool to improve investigation of substantial equivalence in genetically modified organisms: the case of a virus-resistant tomato. Proteomics 4:193-200

Cuyckens F, Claeys M (2004) Mass spectrometry in the structural analysis of flavonoids. J Mass Spectrom 39:1-15

Daniel O, Meier MS, Schlatter J et al (1999) Selected phenolic compounds in cultivated plants: ecologic functions, health implications, and modulation by pesticides. Environ Health Perspect 107:109-114

Delhaize E, Hebb DM, Richards KD et al (1999) Cloning and expression of a wheat (Triticum aestivum L.) phosphatidylserine synthase cDNA. Overexpression in plants alters the composition of phospholipids. J Biol Chem 274:7082-8

Domon B, Costello C (1988) A systematic nomenclature for the carbogydrate fragmentation in FAB-MS/MS spectra of glycoconjugates. Glycoconj J 5:397-409

Eisen MB, Spellman PT, Brown PO et al (1998) Cluster analysis and display of genome-wide expression patterns. Proc Natl Acad Sci USA 95:14863-14868

Finer JJ, Vain P, Jones NW et al (1992) Development of the particle inflow gun for DNA delivery to plant cells. Plant Cell Reports 11:323-328

Ferreres F, Silva BM, Andrade PB et al (2003) Approach to the study of C-glycosyl flavones by ion trap HPLC_PAD-ESI/MS/MS: application to seeds of quince (Cydonia oblonga). Phytochem Anal 14:352-359

Ferreres F, Llorach R, Gil-Izquierdo A (2004) Characterization of the interglycosidic linkage in di-, tri-, tetra- and pentaglycosylated flavonoids and the differentiation of positional isomers by liquid chromatography/electrospray tandem mass spectrometry. J Mass Spectrom 39:312-321

Guess MJ, Wilson SB (2002) Introduction to hierarchical clustering. J Clin Neurophysiol 19:144-151 
Harborne JB (1986) Distribution and function of plant flavonoids. Prog Clin Biol Res Nat 213:15-24

Harborne JB, Grayer RJ (1993) Flavonoids and insects. In: Harborne JB (ed) The flavonoids, advances in Research since 1986. Chapman and Hall, London

Harborne JB, Hall E (1964) Plant polyphenols 12. The occurrence of tricin and of glycoflavones in grasses. Phytochemistry 3:421-428

Harvey MH, McMillan M, Morgan MR et al (1985) Solanidine is present in sera of healthy individuals and in amounts dependent on their dietary potato consumption. Hum Toxicol 4:187-94

Julian EA, Johnson G, Johnson DK et al (1971) The glycoflavonoid pigments of wheat, Triticum eastivum leaves. Phytochemistry 10:3185-3193

Lehesranta SJ, Davies HV, Shepherd LV et al (2005) Comparison of tuber proteomes of potato varieties, landraces, and genetically modified lines. Plant Physiol 138:1690-1699

Markham KR (1982) Techniques of flavonoid identification. Academic Press, London

Obert JC, Ridley WP, Schneider RW et al (2004) The composition of grain and forage from glyphosate tolerant wheat MON 71800 is equivalent to that of conventional wheat (Triticum aestivum L.). J Agric Food Chem 52:1375-1384

Oerke EC, Dehne HW, Schönbeck F et al (1994) Crop production and crop protection. Elsevier Science, Amsterdam

Raychaudhuri S, Stuart JM, Altman RB (2000) Principal components analysis to summarize microarray experiments: application to sporulation time series. In: Altman RB (ed) Pacific symposium on biocomputing 2000, Honolulu, January 2000. Stanford Medical Informatics, Stanford, CA, pp 455-466

Schlaich T, Urbaniak BM, Malgras N et al (2006) Increased field resistance to Tilletia caries provided by a specific anti-fungal virus gene in genetically engineered wheat. Plant Biotechnol J 4:63-75

Shewmaker CK, Sheehy JA, Daley M et al (1999) Seed-specific overexpression of phytoene synthase: increase in carotenoids and other metabolic effects. Plant J. 20:401-412

Shimodaira H (2004) Approximately unbiased tests of regions using multistep-multiscale bootstrap resampling. Ann Statist 32: 2616-2641

Sivamani E, Bahieldin A, Wraith JM et al (2000) Improved biomass productivity and water use efficiency under water deficit conditions in transgenic wheat constitutively expressing the barley HVA1 gene. Plant Sci 155:1-9

Thomas WTB, Baird E, Fuller JD et al (1998) Identification of a QTL decreasing yield in barley linked to Mlo powdery mildew resistance. Mol Breed 4:381-393

Wagner H, Obermeier G, Chari VM et al (1980) FlavonoidC-glycosides from Triticum aestivum L. J Nat Prod 43:583-587

Waridel P, Wolfender JL, Ndjoko K et al (2001) Evaluation of quadrupole time-of-flight tandem mass spectrometry and iontrap multiple-stage mass spectrometry for the differentiation of C-glycosidic flavonoid isomers. J Chrom A 926:29-41

Wiese MV (1991) Compendium of wheat diseases. American Phytopatological Society, St. Paul, MN, U.S.A

Wolfender JL, Hostettmann K (1993) Liquid-chromatographic UV detection and liquid chromatographic thermospray mass-spectrometric analysis of Chironia (Gentianaceae) species - a rapid method for the screening of polyphenols in crude plant-extracts. J Chrom A 647:191-202

Zadoks JC, Chang TT, Konzak CF (1974) A decimal code for the growth stages of cereals. Weed Res 14:415-421 